\title{
QCD Sum Rules in Medium and the Okamoto-Nolen-Schiffer Anomaly
}

\author{
T. Hatsuda, ${ }^{(a)}$ H. Høgaasen, ${ }^{(b)}$ and M. Prakash \\ Department of Physics, State University of New York at Stony Brook, Stony Brook, New York 11794 \\ (Received 9 July 1990)
}

The QCD sum-rule approach for a nuclear medium is developed. The medium dependence of the neutron-proton mass difference calculated from this approach gives effects in nuclei which have direct relevance for the resolution of the Okamoto-Nolen-Schiffer anomaly.

PACS numbers: 21.10.Dr, 12.38.Lg, 14.20.Dh, 21.65.+f

The Okamoto-Nolen-Schiffer (ONS) anomaly ${ }^{1,2}$ is a long-standing problem in nuclear physics. The anomaly is the failure of theory to explain the mass differences $\Delta E$ between mirror nuclei or analog states. Explicitly,

$$
\delta=\Delta E_{\text {expt }}-\Delta E_{\text {th }}
$$

increases with the mass number $A$ and amounts to $\sim 900$ keV for $A=208$. Nuclear structure effects of many kinds have been invoked in the past to solve the problem but without definite success. ${ }^{3}$ A recent proposal invokes mixing effects between $\rho$ and $\omega$ meson exchanges leading to an isospin breaking in nuclear forces that helps to partly reduce the discrepancy. ${ }^{4}$ Other approaches are also possible. In particular, Henley and Krein ${ }^{5}$ have explored the possibility that the resolution of the ONS anomaly might be related to the partial restoration of chiral symmetry in nuclei. By choosing a specific model for chiral-symmetry breaking, viz., the Nambu-JonaLasinio (NJL) model, ${ }^{6}$ and combining it with a nonrelativistic quark model ${ }^{7}$ for the nucleon, they found that the neutron-proton mass difference $\Delta_{n p}=M_{n}-M_{p}$ decreases in the medium as the density of the system increases. Such a decrease bridges the gap between theory and experiment. However, relativistic corrections to the quark model tend to reduce the variation of $\Delta_{n p}$ with nuclear density. ${ }^{8}$ Other models that are successful in hadronic spectroscopy, viz., the MIT bag model and the chiral bag model in their canonical versions, were also found to give similar results. $^{8}$

Since in QCD a partial restoration of chiral symmetry is directly related to a decrease of the quark condensate $\langle\bar{q} q\rangle$ in the medium, it would be better to connect $\Delta_{n p}$ in medium with the vacuum parameters in a model-independent way. This is possible by generalizing the QCD sum rules ${ }^{9}$ to finite density. ${ }^{8}$ The purpose here is to further develop this idea and to point out the relevance of such an approach in resolving the ONS anomaly in a semiquantitative manner.

For the application of QCD sum rules to $\Delta_{n p}$ in vacuum, the operators relevant for the proton and neutron with broken isospin symmetry are

$$
\begin{aligned}
& \Psi_{p}=\epsilon_{a b c}\left[\left(u^{a} C u^{b}\right) \gamma_{5} d^{c}+t\left(u^{a} C \gamma_{5} u^{b}\right) d^{c}\right], \\
& \Psi_{n}=\epsilon_{a b c}\left[\left(d^{a} C d^{b}\right) \gamma_{5} u^{c}+t\left(d^{a} C \gamma_{5} d^{b}\right) u^{c}\right],
\end{aligned}
$$

where $C$ denotes charge conjugation, $a, b$, and $c$ are color indices of the $u$ and $d$ quarks, and $t$ is the mixing strength of the two independent operators having the appropriate symmetry. Following the usual technique of the QCD sum rules, ${ }^{9,10}$ one obtains a formula for the neutron-proton mass difference in vacuum, $\Delta_{n p}$ $\equiv \Delta_{n p}(\delta m, \gamma)$, where $\delta m=m_{d}-m_{u}$ and $\gamma$ is a measure of isospin breaking in the condensates, $\gamma=\langle\bar{d} d\rangle /\langle\bar{u} u\rangle-1$. Since both parameters characterizing isospin breaking are small $\left(\delta m \sim 4 \mathrm{MeV}\right.$ and $\left.\gamma \sim \delta m / \Lambda_{\mathrm{QCD}} \simeq 10^{-2}\right)$, we can safely expand $\Delta_{n p}$ to first order in these parameters. ${ }^{11}$

Calculations of $\Delta_{n p}$ in vacuum based on the Boreltransformed version of the QCD sum rule were performed in Ref. 8 following the approach of Ref. 10. Inclusion of only the continuum contribution does not provide a consistent treatment, ${ }^{12}$ since perturbative $\alpha_{s}$ corrections reduce considerably the absolute value of the nucleon mass in a meaningful range of the Borel parameter. Following the note added in Ref. 10, we adopted the simplest consistent approach, with $t=-1.15$ for the mixing-strength parameter. (We shall return later to discuss other choices for $t$.) For the quark and the gluon condensates, we used the values $\langle\bar{q} q\rangle=(-250 \mathrm{MeV})^{3}$ and $\left\langle\left(\alpha_{s} / \pi\right) G^{2}\right\rangle=(330 \mathrm{MeV})^{4}$, determined by fitting the $J^{p}=\frac{1}{2}^{+}$octet baryons. The isospin-breaking parameter $\gamma$ was extracted by adopting the $\mathrm{SU}_{3}$ breaking parame$\operatorname{ter}^{10}\langle\bar{s} s\rangle /\langle\bar{u} u\rangle-1=-0.3$ and assuming the simple scaling relation $(\langle\bar{d} d\rangle-\langle\bar{u} u\rangle) /(\langle\bar{s} s\rangle-\langle\bar{u} u\rangle) \simeq\left(m_{d}-m_{u}\right) /$ $\left(m_{s}-m_{u}\right)$. This gives $\gamma=-7.8 \times 10^{-3}$, consistent with results of the NJL model, ${ }^{8}$ and also with other independent determinations which yield $\gamma$ in the range -0.006 to $-0.009 .{ }^{13}$ The vacuum value of $\Delta_{n p}=1.3 \mathrm{MeV}$ [after accounting for the electromagnetic contribution of $\simeq-0.76 \pm 0.3 \mathrm{MeV}$ (Ref. 14)] is then obtained with $\delta m=3.75 \pm 0.29 \mathrm{MeV}$.

The above considerations can be generalized to systems with finite baryon densities which are lower than the nuclear matter equilibrium density $\rho_{\mathrm{nm}} \simeq 0.16 \mathrm{fm}^{-3}$. For our purposes here, three essential points from this generalization are as follows: (i) The scalar quark condensate $|\langle\bar{q} q\rangle|$ decreases with density (a signature of partial restoration of chiral symmetry), (ii) the isospinbreaking parameter $\gamma$ varies mildly with density, and (iii) in medium, effects of additional noncovariant condensates in the operator product expansion (OPE), e.g., the vector condensate $\left\langle q^{\dagger} q\right\rangle=\left(N_{c} / N_{f}\right) \rho$, where $\rho$ is the baryon number density, are small for $\Delta_{n p}$. Effects from (iii), not explicitly considered in Ref. 8, keep the pole position of the nucleon propagator nearly constant with density, in contrast to the case when only the scalar condensates are considered. 
Features (i) and (ii) may be explicitly verified by using the NJL model. ${ }^{8}$ In general, $\langle\bar{q} q\rangle=\langle\bar{q} q\rangle_{0}$ - const $\times \rho$, for small $\delta m$ and for $\rho\left\langle\rho_{\mathrm{nm}}\right.$. While $\langle\bar{q} q\rangle$ decreases linearly with density, the quantity $\gamma=\langle\bar{d} d\rangle /\langle\bar{u} u\rangle-1$ $\simeq\langle\bar{d} d\rangle_{0} /\langle\bar{u} u\rangle_{0}-1+O\left(\rho^{2},(\delta m)^{2}, \rho \delta m\right)$ is nearly independent of density at low densities. The density dependence of $\left\langle\left(\alpha_{s} / \pi\right) G^{2}\right\rangle$ may be estimated from the trace anomaly in QCD:

$M_{n}^{(0)}=\left\langle N\left|T_{\mu}^{\mu}-\sum_{q=u, d, s} m_{q} \bar{q} q\right| N\right\rangle=-\frac{9}{8}\left\langle N\left|\left(\alpha_{s} / \pi\right) G^{2}\right| N\right\rangle$.

This leads to

$$
\left\langle\left(\alpha_{s} / \pi\right) G^{2}\right\rangle=\left\langle\left(\alpha_{s} / \pi\right) G^{2}\right\rangle_{0}-\frac{8}{9} M_{n}^{(0)} \rho+O\left(\rho^{2}\right),
$$

where $M_{N}^{(0)}$ is the nucleon mass in the chiral limit. At $\rho_{\mathrm{nm}}$, the decrease of the gluon condensate is less than about $10 \%$.

As for (iii), we have carried out an analysis of the OPE by explicitly including leading-order contributions from operators involving $\alpha_{\mu} \equiv\left\langle\bar{q} \gamma_{\mu} q\right\rangle=\left(\left\langle q^{\dagger} q\right\rangle, 0\right)$. Full details will be published separately. ${ }^{15}$ The vector condensate generates specific contributions to the selfenergy of a nucleon in the medium, i.e., the phenomenological nucleon propagator takes the form

$$
\Pi^{\text {phen }}(q)=\frac{\lambda_{N}}{\left(q_{\mu}-\Sigma_{\mu}^{V}\right) \gamma^{\mu}-\Sigma^{S}},
$$

where $\lambda_{N}$ is the coupling of the operator in Eq. (2) to the nucleon, and $\Sigma^{V}\left(\Sigma^{S}\right)$ is the vector (scalar) self-energy. The OPE side of the sum rule may be written as

$$
\Pi^{\mathrm{OPE}}(q)=q_{\mu} \gamma^{\mu} \Pi_{1}+\Pi_{2}+\alpha_{\mu} \gamma^{\mu} \Pi_{3},
$$

where we regard $\alpha_{\mu}$ as a vector background field, and the polarizations $\Pi_{1,2,3}$ are scalar functions of $\alpha^{2}, q^{2}$, and $\alpha \cdot q$.

The pole position of the propagator is determined by $\left(q_{\mu}-\Sigma_{\mu}^{V}\right)^{2}=\left(\Sigma^{S}\right)^{2}$, which gives $\omega \equiv q_{0}=\Sigma_{0}^{V}(\omega)+\Sigma^{S}(\omega)$ at $\mathbf{q}=0$. As the self-energies are functions of $\omega$, one has to solve a self-consistent equation for the pole position. From the structure of $\Pi_{1,2,3}$ it is, however, easy to see the following: (i) $\Sigma_{0}^{V}$ increases with increasing $\rho$, while $\Sigma^{S}$ decreases with $\rho$ as $\langle\bar{q} q\rangle$ decreases; hence, the pole position $\omega$ stays nearly constant. (ii) The energy difference $\Delta_{n p}=\omega_{n}-\omega_{p}$ is chiefly given by isospin breaking in the scalar part, i.e.,

$$
\Delta_{n p} \simeq \Sigma_{n}^{S}-\Sigma_{p}^{S} \simeq\left[\gamma \frac{8 \pi^{2}\langle\bar{q} q\rangle}{M^{2}}-2(\delta m)\right] f(t),
$$

where $M$ is the optimum value of the Borel mass and $f(t)=2\left(7-2 t-5 t^{2}\right) /\left(5+2 t+5 t^{2}\right)$. For $\Delta_{n p}$, the noncovariant condensate $\left\langle q^{\dagger} q\right\rangle$ appears only in higherdimensional operators, whose effects are suppressed to a large extent as long as the Borel mass $M \sim 1 \mathrm{GeV}$. This is due to isospin symmetry in $N=Z$ systems, i.e., $\left\langle u^{\dagger} u\right\rangle$ $=\left\langle d^{\dagger} d\right\rangle$. To derive Eq. (5), we have taken only the dominant terms on the OPE side, i.e.

$$
\begin{aligned}
\Pi_{1}^{n, p}-- & \left(q^{4} / 64 \pi^{4}\right) \ln \left(-q^{2}\right), \\
\Pi_{2}^{n}-\Pi_{2}^{p}- & -\gamma\left(\langle\bar{q} q\rangle / 4 \pi^{2}\right) q^{2} \ln \left(-q^{2}\right) \\
+ & \left(\delta m / 32 \pi^{4}\right) q^{4} \ln \left(-q^{2}\right) \\
& -\left(\delta m / 2 \pi^{2}\right)(\alpha \cdot q) \ln \left(-q^{2}\right) .
\end{aligned}
$$

The isospin breaking in $\Pi_{3}$ is small compared to that in $\Pi_{2}$ as $\Pi_{3}^{n}-\Pi_{3}^{p}=2\left(\delta m / 3 q^{2}\right)\langle\bar{q} q\rangle$.

Note also that feature (i) above is analogous to the results of a relativistic approach to the nuclear many-body system, ${ }^{16}$ where sizable contributions to $\Sigma^{V}$ and $\Sigma^{S}$ arise from the exchanges of the $\omega$ meson and the $\sigma$ meson, respectively.

The behavior of $M$ vs $\rho$ depends on the details of the Borel sum rule. In the extreme case when $\alpha_{\mu}$ is neglected to fit the OPE side with the phenomenological side, one gets $M=$ const $\times|\langle\bar{q} q\rangle|^{1 / 3}$ for $\rho<\rho_{\mathrm{nm}}$, implying a decrease of $M$ with density. Inclusion of terms with $\alpha_{\mu}$ considerably reduces the density dependence of $M$, since vector contributions to $M$ increase with density. The crucial point for $\Delta_{n p}$ is that while there is a large cancellation between the two terms in Eq. (5), only the first term decreases as $\rho$ increases, as long as the $\rho$ dependence of $M$ is weaker than $|\langle\bar{q} q\rangle|^{2 / 3}$, which is satisfied even in the extreme case considered above. A detailed account of the behavior with the Borel mass will be given in Ref. 15.

The fact that the noncovariant condensates do not significantly affect the structure of $\Delta_{n p}$ in Eq. (5) provides us with a simple form for semiquantitative calculations of the ONS anomaly. Explicitly,

$$
\begin{aligned}
\Delta_{n p} & =C_{1}\left(\langle\bar{q} q\rangle /\langle\bar{q} q\rangle_{0}\right)^{1 / 3}-C_{2} \\
& \simeq\left[5.24\left(\langle\bar{q} q\rangle /\langle\bar{q} q\rangle_{0}\right)^{1 / 3}-3.94\right] \mathrm{MeV},
\end{aligned}
$$

which is valid for $\rho\left\langle\rho_{\mathrm{nm}}\right.$. Above, $\langle\bar{q} q\rangle_{0}$ is the scalar condensate in the vacuum. The numerical values of $C_{1}$ and $C_{2}$ were obtained by the Borel method for $t=-1.15$ as in Ref. 8. In vacuum, Eq. (6) reproduces the correct value of $\Delta_{n p}=1.3 \mathrm{MeV}$. (The electromagnetic contribution is included in the coefficient $C_{1}$.) In medium, the density dependence of $\Delta_{n p}$ is controlled by $\langle\bar{q} q\rangle^{1 / 3}$, and decreases in the low-density region as the condensate decreases.

To see the relevance of this formula to nuclear physics, we need to know how $\langle\bar{q} q\rangle$ varies in the nuclear medium. Since lattice QCD results for $\langle\bar{q} q\rangle$ in medium are not yet available, we turn to the trends found in effective field theories that probe the structure of the vacuum. ${ }^{6,17,18}$ In such theories, the quark condensate decreases linearly with density for $\rho \leq \rho_{\mathrm{nm}}$, although the precise rate of decrease is somewhat dependent on the parameters chosen. From dimensional arguments, the behavior of the dynamical mass of the NJL model in medium can be identified with that of $\langle\bar{q} q\rangle^{1 / 3}$ of the QCD sum rule with 
the result

$$
\langle\bar{q} q\rangle^{1 / 3} /\langle\bar{q} q\rangle_{0}^{1 / 3}=1-C \rho / \rho_{0}=G(\rho),
$$

where $C$ lies in the range $0.1-0.2$.

We turn now to consider (nearly isosymmetric) nuclei, where in one case there is a neutron, and in the other there is a proton, outside a common core. As a zeroorder approximation, we take $P_{A}(r)=\rho_{A}(r)-\rho_{A-1}(r)$ for the probability distribution of the last nucleon which is moving in a nuclear medium with $\rho_{A-1}(r)$. Comparing the masses of the two mirror or analog nuclei, the medium-dependent $\Delta_{n p}$ in Eq. (6) will give a contribution

$$
\left(\Delta_{n p}\right)_{\text {nuclei }}=\int\left[C_{1} G\left(\rho_{A-1}\right)-C_{2}\right] P_{A}(r) d^{3} r .
$$

Use of the local-density approximation is implicit in the calculation of $G\left(\rho_{A-1}\right)$. Equation (8) leads to "an anomaly of the ONS type" of the size $\delta=\left(\Delta_{n p}\right)_{0}$ $-\left(\Delta_{n p}\right)_{\text {nuclei, }}$, and is given by

$$
\delta=C_{1}\left[1-\int G\left(\rho_{A-1}\right) P_{A}(r) d^{3} r\right] \equiv C_{1} D .
$$

Note that $\delta$ depends on the mass number $A$, and $C_{2}$ is irrelevant in the final formula. To account for the uncertainties in the value of $\gamma(=-0.006$ to -0.009$)$ and the electromagnetic contribution $\simeq \pm 0.3 \mathrm{MeV},{ }^{14}$ we attach a $\pm 26 \%$ error on $C_{1}$, i.e., $C_{1}=5.24 \pm 1.36 \mathrm{MeV}$.

In Table I, we show how $\delta$ varies with $A$ using $C_{1}$ $=5.24 \pm 1.36 \mathrm{MeV}$ and $C=0.2$. Results for lower values of $C$ simply scale with its magnitude. The density distributions occurring in Eq. (9) were calculated using a Woods-Saxon potential with parameters chosen to fit the experimental rms values of the core densities and the single-particle energies of the valence particle. The calculated $\delta$ varies mildly with mass number $A$. Noticeable shell effects arising from the differences in the average density felt by a nucleon outside a closed shell compared to a nucleon within a close shell are also evident. The numbers are of the right order of magnitude to partly resolve the ONS anomaly. For comparison, the "empirical" ONS discrepancy from different sources $2,3,19$ is also given. The spread in the empirical values reflects the uncertainties in using different models of nuclear structure and nuclear forces in the extraction of the discrepancy.

TABLE I. Comparison of $\delta$ from Eq. (9) with typical "empirical" ONS discrepancies. The two values from Ref. 19 correspond to results based on the density-matrix expansion and the Skyrme II forces, respectively. The prediction of the QCD sum rules correspond to $C_{1}=5.24 \pm 1.36 \mathrm{MeV}$.

\begin{tabular}{lcccc}
\hline \hline & & \multicolumn{2}{c}{$\delta(\mathrm{MeV})$} \\
Nuclei & Eq. (9) & Ref. 2 & \multicolumn{1}{c}{ Ref. 3 } & Ref. 19 \\
\hline${ }^{15} \mathrm{O}-{ }^{15} \mathrm{~N}$ & $0.53 \pm 0.14$ & $\ldots$ & $0.16 \pm 0.04$ & $(0.29)(0.38)$ \\
${ }^{17} \mathrm{~F}-{ }^{17} \mathrm{O}$ & $0.29 \pm 0.07$ & 0.21 & $0.30 \pm 0.06$ & $(0.19)(0.30)$ \\
${ }^{39} \mathrm{Ca}^{39} \mathrm{~K}$ & $0.57 \pm 0.15$ & $\ldots$ & $0.22 \pm 0.08$ & $(0.43)(0.54)$ \\
${ }^{41} \mathrm{Sc}-{ }^{41} \mathrm{Ca}$ & $0.42 \pm 0.11$ & 0.62 & $0.5 \pm 0.10$ & $(0.35)(0.44)$ \\
\hline \hline
\end{tabular}

In view of this, definite conclusions cannot be drawn from a comparison with the calculated shell effects. It is also possible that our calculations include only a part of the shell effects.

Note that our results for $\delta$ are chiefly given by the quantity $C_{1}$, which is associated with chiral restoration, with modulations dependent on nuclear structure inputs [the quantity $D$ in Eq. (9)]. In light of this, it appears that the ONS anomaly could be a striking signal of chiral restoration in nuclei. In essence, the partial restoration of chiral symmetry makes the proton and neutron isosymmetric at low densities. This explanation of the ONS anomaly is quite distinct from most other attempts. Traditionally, one invokes charge-symmetry breaking for the resolution of the anomaly; here it is the restoration of chiral symmetry that furnishes (at least partly) the resolution of this long-standing problem.

We now examine the degree to which results from Eq. (5) are sensitive to the mixing parameter $t$. Although Eq. (6) has been obtained with $t=-1.15$, favored by the Borel-transform method, similar formulas may be obtained purely from dimensional reasons for a wide class of such currents, i.e., other values of $t$. In fact, the forms of $\Psi(x)$ favored in the literature all give $\Delta_{n p}$ $=\left(\alpha_{1}-\alpha_{2} \delta m\right) f(t)$, where $\alpha_{2}$ is a positive dimensionless constant. The correct sign of $\Delta_{n p}$ is obtained by a positive value of $\alpha_{1}$, which has the dimension of mass [and $f(t)>0$ ]. Of the two quantities, $|\langle\bar{q} q\rangle|^{1 / 3}$ and $\left\langle G^{2}\right\rangle^{1 / 4}$, having the dimension of mass, the former chiefly determines the magnitude of $\alpha_{1}$. It therefore follows that $\Delta_{n p}$ decreases in medium as the condensate decreases. All these forms are therefore of the correct structure to be relevant for the resolution of the ONS anomaly. To illustrate our point and to see the effect of the continuum, we now derive an exact formula for the hadronic contribution of $\Delta_{n p}$. For the most used choice $t=-1$ (due to Ioffe ${ }^{9}$ ) for which $f(t)=1$, we find

$$
\Delta_{n p}=-A_{1} \gamma M_{0}-A_{2} \delta m,
$$

with

$$
\begin{aligned}
& A_{1}=1+4 a M_{0} / 3 y^{2} z_{2}, \\
& A_{2}=2+\frac{M_{0}}{y^{2} z_{2}}\left[\left(M_{0}-\frac{b}{a}\right) y z_{1}-2 a\right],
\end{aligned}
$$

where $z_{1}=1-\exp \left(-s_{0} / y\right), \quad$ and $\quad z_{2}=1-\left(1+s_{0} / y\right)$ $\times \exp \left(-s_{0} / y\right)$. Above, $M_{0} \equiv M_{0}(y)$ and $y$ are the nucleon mass in the chiral limit and the Borel mass squared, respectively. $s_{0}$ is the continuum threshold, assumed here for clarity to be the same for the neutron and the proton, and $a \equiv-(2 \pi)^{2}\langle\bar{q} q\rangle$ and $b \equiv \pi^{2}\left\langle\left(\alpha_{s} / \pi\right) G^{2}\right\rangle$.

As before, we use the values $\langle\bar{q} q\rangle=(-250 \mathrm{MeV})^{3}$ and $\left\langle\left(\alpha_{s} / \pi\right) G^{2}\right\rangle=(330 \mathrm{MeV})^{4}$. Using $s_{0}=1.9 \mathrm{GeV}^{2}$, we find the following results: (i) For $y=1.7 \mathrm{GeV}^{2}, M_{0}=903.5$ $\mathrm{MeV}, A_{1}=1.84$, and $A_{2}=1.58$, and (ii) for $y=2.7$ $\mathrm{GeV}^{2}, M_{0}=939.2 \mathrm{MeV}, A_{1}=1.68$, and $A_{2}=1.83$. The 
gluon contributions to $A_{2}$ are roughly $\frac{1}{5}$ of the quarkcondensate contributions. In a picture where both the quark and gluon condensates are being driven to their perturbative values, $A_{2}$ might be expected to vary slowly with density. This provides a plausibility argument for the physics content of Eqs. (5) and (6). These numerical values then lead to a form for $\Delta_{n p}$ in medium similar to Eq. (6), but with somewhat larger coefficients, which is expected from the approximate formula in Eq. (5). If we keep $\delta m \simeq 4 \mathrm{MeV}$ as before and choose $\gamma(=-5.46$ $\times 10^{-3}$ ) at the lower end of our previous estimate, we obtain $\Delta_{n p}=\left[8.6\left(\langle\bar{q} q\rangle /\langle\bar{q} q\rangle_{0}\right)^{1 / 3}-7.3\right] \mathrm{MeV}$. With these coefficients, Eq. (9) gives somewhat larger values of $\delta$ than given by Eq. (6). Note that in keeping $t$ fixed at its vacuum value, one hopes that a perturbative approach may suffice to predict physical effects at low density. At high densities, self-consistent procedures including the role of higher-dimensional operators may have to be developed to check if in-medium modifications of $t$ are not large.

We have verified that effects of charge-symmetry breaking in the continuum do not affect the general form of Eq. (6), by performing an analysis using finite-energy sum rules ${ }^{20}$ with different thresholds $s_{0}$ for the neutron and the proton. Furthermore, we note from the results of Ref. 12 that $\alpha_{s}$ corrections leave the generic form of Eq. (6) intact, as these corrections do not change the mass ratios in the same flavor multiplets, although they decrease the particle masses. As with the Boreltransform method, we again find the coefficient $C_{1}$ to be larger than that in Eq. (6), which seems adequate to resolve the ONS anomaly (see Table I).

Our general conclusions are as follows. For a wide class of nucleon currents, the neutron-proton mass difference can be written as the difference of two fairly large positive terms, of which only one varies strongly with $\langle\bar{q} q\rangle^{1 / 3}$. Any variation of $\langle\bar{q} q\rangle^{1 / 3}$ in medium will therefore lead to an enhanced relative variation of $\Delta_{n p}$. If higher-order effects do not change this trend, ${ }^{21}$ this QCD sum-rule result is of direct relevance for the resolutin of the ONS anomaly. A few directions for further study are suggested: (i) There is a need to bring the "empirical ONS discrepancy" under better control by improved nuclear structure calculations. (ii) For $N \neq Z$ systems such as ${ }^{208} \mathrm{~Pb}$, the role of finite-density effects in QCD sum rules requires further investigation. Further efforts in reassessing the various effects suggested in the literature may help in the final resolution of the ONS anomaly.

We thank Gerry Brown, Hans Hansson, and Ismail Zahed for discussions and encouragement. Since the submission of this work, we have received a few preprints $^{22,23}$ dealing with related material. This work was supported by the U.S. Department of Energy under Grant No. DE-FG02-88ER40388. (a) Present address: Institute for Nuclear Theory, HN-12, University of Washington, Seattle, WA 98195.

(b) Permanent address: Department of Physics, University of Oslo, P.O. Box 1048 Blindern, N-0316, Oslo 3, Norway.

'K. Okamoto, Phys. Lett. 11, 150 (1964).

${ }^{2}$ J. A. Nolen, Jr., and J. P. Schiffer, Annu. Rev. Nucl. Sci. 19, 414 (1969).

${ }^{3}$ S. Shlomo, Rep. Prog. Phys. 41, 957 (1978).

${ }^{4}$ P. G. Blunden and M. J. Iqbal, Phys. Lett. B 198, 14 (1987).

${ }^{5}$ E. M. Henley and G. Krein, Phys. Rev. Lett. 62, 2586 (1989).

${ }^{6}$ Y. Nambu and G. Jona-Lasinio, Phys. Rev. 122, 345 (1961); 124, 246 (1961); T. Hatsuda and T. Kunihiro, Prog. Theor. Phys. 74, 765 (1985).

${ }^{7}$ N. Isgur, Phys. Rev. D 21, 779 (1980).

${ }^{8}$ T. Hatsuda, H. Høgaasen, and M. Prakash, Phys. Rev. C 42, 2212 (1990).

${ }^{9}$ A. Shifman, A. I. Vainshtein, and V. I. Zakharov, Nucl. Phys. B147, 385 (1979); B. L. Ioffe, Nucl. Phys. B188, 317 (1981); V. M. Belyaev and B. L. Ioffe, Zh. Eksp. Teor. Fiz. 84, 1236 (1983) [Sov. Phys. JETP 57, 716 (1983)]; L. J. Reinders, H. Rubinstein, and S. Yazaki, Phys. Rep. 127, 1 (1985).

${ }^{10}$ D. Espriu, P. Pascual, and R. Tarrach, Nucl. Phys. B214, 285 (1983); see the note added in this paper for the error is sign of the gluon condensate in previous calculations.

${ }^{11}$ Note that a perturbative treatment is not always valid for the octet-breaking case, e.g., when $\delta m=m_{s}-m_{u} \sim 150 \mathrm{MeV}$ and $\gamma=\langle\bar{s} s\rangle /\langle\bar{u} u\rangle-1 \sim-0.3$.

${ }^{12}$ A. A. Ovchinnikov, A. A. Pivovarov, and L. R. Surguladze, Yad. Fiz. 48, 562 (1988) [Sov. J. Nucl. Phys. 48, 358 (1988)].

${ }^{13}$ N. Paver, Riazuddin, and M. D. Scadron, Phys. Lett. B 197, 430 (1987); S. Narison, Phys. Lett. B 216, 191 (1990).

${ }^{14}$ The electromagnetic $n-p$ mass difference calculated using the Cottingham formula gives $-0.76 \pm 0.30 \mathrm{MeV}$ [cf. J. Gasser and H. Leutwyler, Phys. Rep. 87, 77 (1982)].

${ }^{15}$ T. Hatsuda, H. Høgaasen, and M. Prakash (to be published).

${ }^{16}$ B. D. Serot and J. Walecka, in Advances in Nuclear Physics, edited by J. W. Negele and E. Vogt (Plenum, New York, 1985), Vol. 16.

${ }^{17}$ T. Hatsuda and T. Kunihiro, Phys. Rev. Lett. 55, 158 (1985); Phys. Lett. B 185, 304 (1987); V. Bernard, U.-G. Meissner, and I. Zahed, Phys. Rev. Lett. 59, 966 (1987).

${ }^{18}$ T. L. Ainsworth, G. E. Brown, W. Weise, and M. Prakash, Phys. Lett. B 200, 413 (1988).

${ }^{19}$ H. Sato, Nucl. Phys. A296, 378 (1976).

${ }^{20}$ N. V. Krasnikov, A. A. Pivovarov, and N. N. Tavkhelidze, Z. Phys. C 19, 301 (1983).

${ }^{21}$ Effects of higher-dimensional operators remain an open problem in QCD sum rules, even at zero density; see, e.g., $\mathrm{H}$. G. Dosch, M. Jamin, and S. Narison, Phys. Lett. B 220, 251 (1989).

${ }^{22}$ C. Adami and G. E. Brown, State University of New York Report No. SUNY-NTG-90-38, 1990 (to be published).

${ }^{23}$ T. D. Cohen, R. J. Furnstahl, and M. Banerjee, Phys. Rev. C 43, 357 (1991); T. D. Cohen, R. J. Furnstahl, and D. K. Griegel, University of Maryland Report No. UMPP 91-177, 1991 (to be published). 